\title{
CONSTANT FINAL ENERGY AND CONSTANT INITIAL ENERGY SPECTROSCOPY (*)
}

\author{
G. J. LAPEYRE, R. J. SMITH, J. KNAPP $\left({ }^{* *}\right)$ and J. ANDERSON \\ Physics Department, \\ Montana State University, \\ Bozeman, Montana 59717, U.S.A.
}

\begin{abstract}
Résumé. - La correspondance existant entre l'émission photoélectronique et l'énergie des photons révèle des informations fondamentales sur les propriétés de la matière. L'emploi de sources synchrotrons permettant de faire varier de façon continue $h v$, assure un accès direct à cette correspondance. Deux méthodes de variation de $h v$ ont été utilisées. La première. appelée Spectre à énergie Finale Constante (CFS), consiste à maintenir constante l'énergie d'analyse $E_{\mathrm{f}}$ du spectromètre à photoélectron et à faire varier $h v$. Dans la seconde méthode, $E_{\mathrm{f}}$ et $h v$ varient de façon synchrone de telle sorte que $E_{\mathrm{f}}-h v$ demeure constant. Puisque $E_{\mathrm{f}}-h v$ est l'énergie initiale $E_{\mathrm{l}}$ pour les excitations à une particule, la courbe obtenue est appelée Spectre à énergie Initiale Constante (CIS). Les spectres CFS, CIS et la traditionnelle Courbe de Distribution Energétique (EDC) constituent les trois méthodes utilisées en spectroscopie des photoélectrons.

Les méthodes CFS et CIS ont été appliquées à l'étude des excitations à partir des niveaux de valence et de coeur, et à celle des propriétés de volume et de surface, pour des solides et des gaz. La méthode CIS est une spectroscopie de l'état final puisque $E_{\mathrm{i}}$ est fixe pendant le balayage en $E_{\mathrm{f}}$, ce qui permet, par exemple, la détermination des énergies des bandes de conduction et des mécanismes de déclin du trou lors d'une excitation des niveaux de cœur. En particulier, le comportement au seuil d'un niveau de cœur peut être étudié et les résultats obtenus pour les transitions entre les niveaux $3 \mathrm{~d}$ de GaAs vers l'état excitonique vide de surface et le déclin subséquent du trou de cœur sont présentés. Avec le développement de la photoémission résolue angulairement, les méthodes CFS et CIS ont trouvé de nouvelles applications : par exemple, la structure observée dans l'émission directe des niveaux de cœur $4 \mathrm{~d}$ de In dans InP a été attribuée aux bandes de conduction. La conservation de la composante parallèle du vecteur d'onde $\mathbf{k}_{\|}$est la règle de sélection principale pour la spectroscopie en photoémission résolue angulairement et fonction de la polarisation. Puisque la méthode CFS fixe $E_{\mathrm{f}}$, un spectre CFS, obtenu à un angle polaire particulier, détermine les propriétés d'émission pour une valeur unique de $\mathbf{k}_{||}$ce qui facilite l'interprétation. Cet exposé sera donc consacré aux différents types d'études utilisant les méthodes CFS et CIS. Enfin les résultats récents, - énergies des points critiques des bandes de valence et de conducteur de GaAs et point $\mathrm{L}_{1}$ du Ni déterminant une nouvelle largeur de la bande $\mathrm{d}$ - obtenus à partir de données collectées au synchrotron du Wisconsin sont présentés.
\end{abstract}

Abstract. - The photon energy dependence of photoelectron emission contains significant information about the properties of matter. By taking advantage of the synchrotron radiation continuum one can scan the photon energy $h v$ and directly obtain this photon energy dependence. We have developed and used two modes of scanning $h v$. In one the kinetic energy window of the photoelectron spectrometer, $E_{\mathrm{f}}$, is held constant as $h v$ is varied and is referred to as the Constant Final-energy Spectrum (CFS). For the other $E_{\mathrm{f}}$ and $h v$ are synchronously varied such that $E_{\mathrm{f}}-h v$ is constant. Since $E_{\mathrm{f}}-h v$ is the initial energy $E_{1}$ for one particle excitations, the resultant curve is termed the Constant Initial-energy Spectrum (CIS). The CFS and the CIS together with the traditional Energy Distribution Curve (EDC) form the three modes of photoemission spectroscopy. The CFS and CIS modes have been applied to the study of valence and core state excitations, surface and volume properties, for solids as well as gases. The CIS provides one with a final state spectroscopy since $E_{\mathrm{i}}$ is fixed as $E_{\mathrm{f}}$ is scanned which results for example in the determination of conduction band energies for valence excitation and hole decay mechanisms for core excitations. In particular the behaviour at the core threshold can be studied and the results for the GaAs 3d core-to-empty-surface-

(*) Research sponsored by the U.S. Air Force Office of Scientific Research, Air Force Systems Command under Grant No. 752872 and contract No. F49620-77-C-0125. The Synchrotron Centre is supported by NSF Grant No. DMR-74-15089.
(**) Present address : IBM Thomas J. Watson Research Centre, Yorktown Heights, N. Y. 10598, U.S.A. 


\begin{abstract}
state exciton and the ensuing core hole decay processes are presented. With the development of angle-resolved photoemission the CFS and CIS modes have found new applications. Structure in the direct emission of the In $4 d$ core of InP due to conduction band states is presented. A principal selection rule in polarization-dependent, angle-resolved photoemission spectroscopy (PARUPS) is conservation of the parallel component of the wave vector, $\mathbf{k}_{\|}$. Since the CFS mode fixes $E_{\mathrm{f}}$, a CFS measured at a particular polar angle determines the emission properties for a unique $\mathbf{k}_{\|}$value which facilitates the interpretation. An overview of the various types of studies performed with the CFS and CIS modes will be given. Recent results obtained from data collected at the Wisconsin Synchrotron Radiation Centre are presented for valence band and conduction band critical point energies of GaAs and for the $L_{1}$ point of $\mathrm{Ni}$ which determines a new d-band width.
\end{abstract}

1. Introduction. - The standard method of doing Ultraviolet Photoemission Spectroscopy (UPS) has been to take Energy Distribution Curves (EDC's) the number of emitted electrons, $N\left(E_{\mathrm{f}} ; h v\right.$ fixed), as a function of final-state energy, $E_{\mathrm{f}}$, at constant photon energy, $h v$. The kinetic energy of the emitted electron is $E_{\mathrm{f}}$ minus the vacuum threshold energy. A family of such EDC's at closely spaced $h v$ values could map out the emission surface $N\left(E_{\mathrm{f}}, h v\right)$. In principle, all the information available from the photoemissive properties of the sample for a given geometry are implicit in these data. In practice, however, detailed amplitude differences in adjacent EDC's are difficult or impossible to extract. «We have therefore taken advantage of the smooth stable synchrotron radiation continuum and the possibility of sweeping $h v$ continuously, and we have devised two new modes of experimental procedures for UPS " [1]. These modes scan the photon energy such that a Constant Final energy Spectrum (CFS) or a Constant Initial energy Spectrum (CIS) is measured, and together with the EDC form the three modes of UPS. The first experiment which measured a photocurrent while sweeping the synchrotron continuum was the yield study by Haensel et al. [2]. The investigators did not use an energy window as in UPS but they did use selected biases on the collector to determine partial yields resulting from electrons with sufficient kinetic energy to surmount the bias. «In the CFS and CIS modes, structure in the $N\left(E_{\mathrm{f}}, h v\right)$ surface which is difficult to extract, or even unobservable in a family of EDC's, is readily apparent in a single experimental run. Hence these modes greatly facilitate the observation and characterization of 1) initial and final state structures for the electronic transitions, 2) optical selection rules and/or matrix element effects, 3) inelastic scattering, 4) Auger transitions and 5) many-body effects, i.e. excitons, plasmons, two-electron excitations, etc. A study of these properties can be carried out for either interband or core to conduction band continuum transitions " [1].

The three modes of UPS have found wide applicability in the study of ionic insulators [3-6], metals [710], semi-conductors [11-17], layered crystals [18, 19], gases [20], intrinsic surfaces $[8,11,19]$, and chemisorbed surfaces $[2,21,22]$. In executing an experimental study we find it useful to intermingle EDC,
CIS, and CFS data collection to gain a full perspective of a sample's photoemissive features. With the development of angle-resolved UPS, the CFS and CIS have continued to be important modes of data collection. In this paper we will give examples of electronic structure studies with valence electron excitation (Sec. 2), core electron excitation (Sec. 3.1), and core hole decay (Sec. 3.2). The data were collected at the Wisconsin Synchrotron Radiation Centre. First a brief introduction of angle-resolved, CFS and CIS concepts is given.

$P A R U P S$ : Because synchrotron radiation is polarized, one by necessity does polarization-dependent, angle-resolved UPS (PARUPS) at a synchrotron centre. The observed polarization effects are very strong and can provide initial state symmetry data $[8,14]$. Polarization effects are observed even with unpolarized resonance lamp radiation when the angle of incidence is varied, because the ratio of $p$ to $s$ polarization for the absorbed flux changes [23]. For a given photon energy and incident geometry of the vector potential, $\mathbf{A}\left(\theta_{A}, \varphi_{A}\right)$, one measures the emission into a small energy window, $\Delta E$, at $E_{k}$, and into a small solid angle at $\theta_{p}$ and $\varphi_{p}$ which determines the momentum of the emitted electron, $p^{2}=2 m E_{k}$. The normal direction is $\theta_{p}=0$ and the azimuth $\varphi_{p}$ is referenced to a crystal symmetry axis. For our spectrometer, $\Delta E$ is about 0.5 or about $0.35 \mathrm{eV}$ and the solid angle is $\pm 2^{\circ}$ or $\pm 6^{\circ}$. The spectrometer configurations are described elsewhere $[8$, $14,24]$. The angle-resolved selection rule is that the parallel component of the wave vector is conserved when an electron crosses the solid-vacuum interface; $\mathbf{p}_{\|}=h \mathbf{k}_{\|\|}$[25]. There are no general, simple relationships between the perpendicular component of the momentum inside and outside of the solid. The CIS and CFS modes, as presented in sections 2 and 3 , can be used to take special advantage of the angleresolved selection rule.

$C F S$ : By scanning $h v$ and holding fixed the energy window of the electron spectrometer, one measures the CFS, $N\left(h v, E_{\mathrm{f}}\right.$ fixed) [26]. Using energy conservation for one particle excitations, $h v=E_{\mathrm{f}}-E_{\mathrm{i}}$, one can replot the CFS as $N\left(-E_{\mathrm{i}} ; E_{\mathrm{f}}\right.$ fixed). By examining a family of CFS's, one can classify a CFS peak as an initial energy peak if the peak has essentially a given $E_{\mathrm{i}}$ value, or a photon energy peak if it has 
a given $h v$ value. This classification is analogous to the traditional technique of plotting families of EDC's against $E_{\mathrm{f}}$ and then $E_{\mathrm{i}}$ to determine if an EDC peak has $E_{\mathrm{f}}$ or $E_{\mathrm{i}}$ character. Having the final energy fixed in the CFS has several advantages; for example, one observes how various different initial states couple to the same final energy, and for angle-resolved spectroscopy, fixing $E_{\mathrm{f}}$ makes $\mathbf{k}_{\|}$a constant for the spectrum.

$C I S$ : By synchronously scanning $h v$ and $E_{\mathrm{f}}$ so that $h v-E_{\mathrm{f}}$ is held fixed, one measures the CIS, $N\left(h v, h v-E_{\mathrm{f}}\right.$ fixed). Using energy conservation, the CIS can be replotted as $N\left(E_{\mathrm{f}}, E_{\mathrm{i}}\right.$ fixed), and in analogy to the discussion in the above paragraph, CIS peaks may be classified as final energy peaks or photon energy peaks [5]. Hence the CIS mode directly provides one with a final state spectroscopy which has been used to study many kinds of materials.

A new application of the CIS and CFS with significant potential is in the determination of surface EXAFS (Extended X-ray Absorption Fine Structure) since such data may provide structural information. This application comes about because the photocurrent is related to the photoabsorption. Of the possible methods of achieving such measurements, two are of particular interest; one is to measure a CFS on an Auger line, and the other is to measure the direct core emissions with a CIS, either of which can be made surface sensitive by detecting photoemission which originates only from an adsorbate core level, or from core levels on atoms restricted to sites near the surface as, for example, in the layered compounds. P. A Lee has calculated CFS spectra for Auger emission from Se chemisorbed on several faces of nickel in various adsorption sites, and discusses the relationship of CIS spectra to EXAFS for direct core emission [27].

2. Valence band emission studies. - 2.1 NORMAL EMISSION GEOMETRY. - Photoemission measurements which utilize the continuum of synchrotron radiation in a photon energy scanning mode make it possible to separately study the initial and final states involved in the photoexcitation process. The analysis of such spectra in terms of three-dimensional bulk band structure is quite difficult, but can be greatly simplified by using angle-resolved measurements. This simplification results from the fact that electron momentum parallel to the sample surface, $\mathbf{k}_{\|}$, is conserved for electron emission into vacuum [25]. For two-dimensional systems, a determination of $E_{\mathrm{i}}$ vs $\mathbf{k}_{\| !}$will uniquely determine the band structure, but for three-dimensional systems, the non-conservation of $\mathbf{k}_{\perp}$ means that all transitions between states along a line in the Brillouin Zone (BZ) which is perpendicular to the surface must be considered. Thus if $\mathbf{k}_{\|}$is determined by a measurement, only bands along that single $\mathbf{k}_{\|}$line have to be analysed rather than all the bands in the $\mathrm{BZ}$ volume. Since $\mathbf{k}_{\|}$is the parameter which specifies the particular line of $\mathbf{k}_{\perp}$ values, we use the term « $\mathbf{k}_{\|}$line » to indicate that line in the $\mathrm{BZ}$ which is parallel to the surface normal at a distance, $\left|\mathbf{k}_{\|}\right|$, from the centre of the BZ at an azimuth, $\hat{k}_{\|}$.

The most direct way to study a single line in $k$-space is to collect only those photoelectrons at normal exit angles, i.e. perpendicular to the surface $[7,8,28]$. The normally emitted electron has $\mathbf{k}_{\|}=0$. For the (110) cleavage face of GaAs the line with $\mathbf{k}_{\|}=0$ is the $\Sigma$ line from $\Gamma$ to $\mathrm{K}$ to $\mathrm{X}$ in the $\mathrm{BZ}$. In figure 1

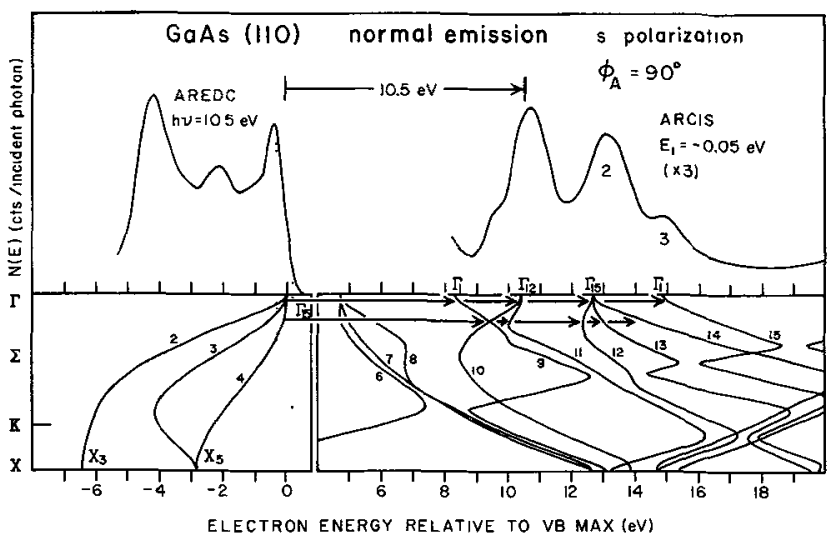

FIG. 1. - Normal emission ARCIS and AREDC from GaAs (110) showing transitions which originate near the VB maximum. Bands along $\Sigma$ are from reference [29].

we show an AREDC referenced to initial energy, $E_{\mathrm{i}}$, and an ARCIS referenced to final energy, $E_{\mathrm{f}}$, plotted alongside the energy bands for the $\Sigma$ line [29]. The position $\left(k_{\perp}\right)$ of a direct transition along this line must be determined from energy considerations since $\mathbf{k}_{\perp}$ is not conserved. The initial energy parameter for the ARCIS in figure 1 is chosen to be at the Valence Band (VB) maximum which thus restricts the transitions to those in a small $k$-space volume about $\Gamma$, consistent with any selection rules. The three peaks observed at 10.6, 12.8, and $14.9 \mathrm{eV}$ agree very well with the values calculated by Pandey and Phillips [29] of 10.4, 12.6, and $14.9 \mathrm{eV}$ for the $\Gamma_{12}$, $\Gamma_{15}$ and $\Gamma_{1}$ points respectively.

The restriction to normal emission means that all three modes of photoemission measure transitions along $\Gamma$. The ARCIS will measure how initial states at a fixed initial energy couple to the final states along $\Sigma$, while the ARCFS will measure the spectrum of initial states which couple to final states at a specified energy. The AREDC is a sum of all transitions between those states separated by a fixed $h v$ and thus may contain both initial and final state effects. To demonstrate the initial state character of the ARCFS we show in figure 2 a normal emission ARCFS for cleaved $\mathrm{GaAs}(110)$, obtained with $E_{\mathrm{f}}=9.4 \mathrm{eV}$, plotted alongside the $\Sigma$ bands as a function of initial energy, with the upper scale showing photon energy. This final energy is chosen for display 


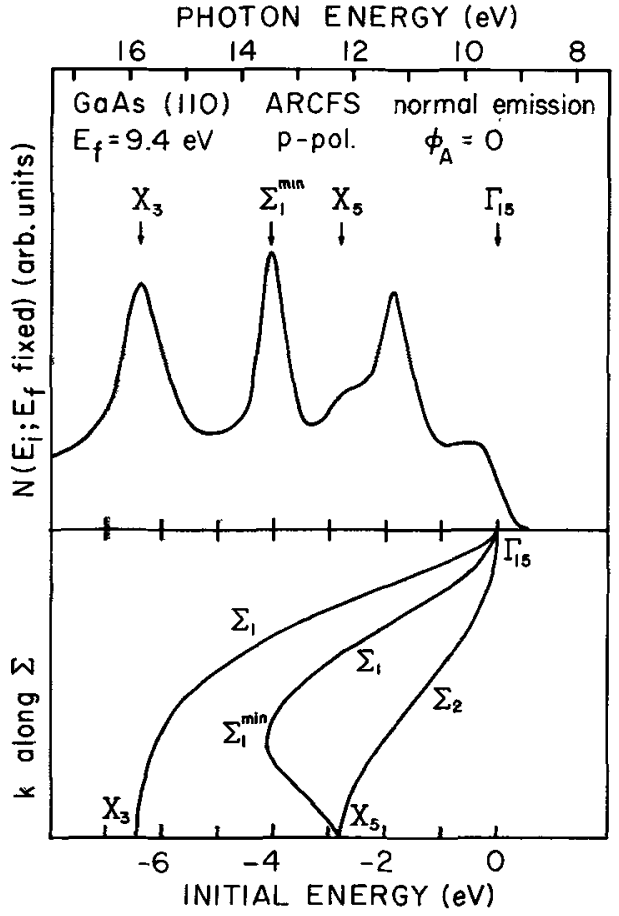

FIG. 2. - Normal emission ARCFS from cleaved GaAs (110) exhibiting several critical points in the VB along $\Sigma$.

since transitions from small $k$-space volumes near several critical points are observed due to the multiplicity of final state bands at $9.4 \mathrm{eV}$ (see Fig. 1). The leading shoulder is attributed to the VB maximum at $\Gamma_{15}$. The feature at $-1.8 \mathrm{eV}$ is due to a high density of final states, as determined by examining a family of ARCFS's, while the features at -2.8 and -4.1 are attributed to transitions near $X_{5}$ and $\Sigma_{1 \text { min }}$ respectively. The deeper peak at $-6.3 \mathrm{eV}$ is assigned to an excitation near $\mathrm{K}$ since no final bands exist at $\mathrm{X}$ for $\mathrm{E}_{\mathrm{f}}=9.4 \mathrm{eV}$. However, ARCFS's taken for different $E_{\mathrm{f}}$ values show this peak moving to deeper $E_{\mathrm{i}}$, giving $E_{\mathrm{i}}=-6.8 \mathrm{eV}$ for $\mathrm{X}_{3}$.

2.2 NON-NORMAL EMISSION GEOMETRY. - For measurements at non-normal emission angles, the three modes of photoemission measurement take on new meaning. As already noted, the important quantity is conservation of parallel wave vector,

$$
\left|\mathbf{k}_{\|}\right|=\sin \theta_{p} \sqrt{E_{k} /\left(h^{2} / 2 m\right)} .
$$

Hence if emission is measured at fixed $\theta_{p}$, the AREDC and ARCIS modes sweep through a range of $\mathbf{k}_{\|}$ values because $E_{k}$ is changing. For this reason we say that transitions in the non-normal AREDC and ARCIS represent contributions from a halfplane normal to the surface in the BZ, when the data is analysed using the bulk, three-dimensional band picture. A one-dimensional BZ analysis can still be made for each peak in a spectrum, but the bands related to different peaks will lie on different $\mathbf{k}_{\|}$ lines. For the ARCFS, however, $E_{k}$ is a constant, so $\mathbf{k}_{\|}$is constant and a one-dimensional analysis for the whole ARCFS spectrum can be made using the bands for a single $\mathbf{k}_{\|}$line. The single $\mathbf{k}_{\|}$line can be arbitrarily chosen to be a symmetry line which is not accessible using the normal emission techniques as described above. An example of the ARCFS mode used for an off-normal symmetry line analysis is shown in figure 3, where emission from the GaAs (110) face is restricted to the $\mathrm{Q}$ line. The line $\mathrm{Q}$ runs from $L$ to $W$ on the hexagonal face of the $B Z$ and since it does not pass through the zone centre, this line is not accessible using normal emission techniques $\left(\mathbf{k}_{\|} \neq 0\right.$ for Q). The ARCFS in figure 3 was obtained with $E_{\mathrm{f}}=12.3 \mathrm{eV}, \theta_{p}=44.5^{\circ}$ in a

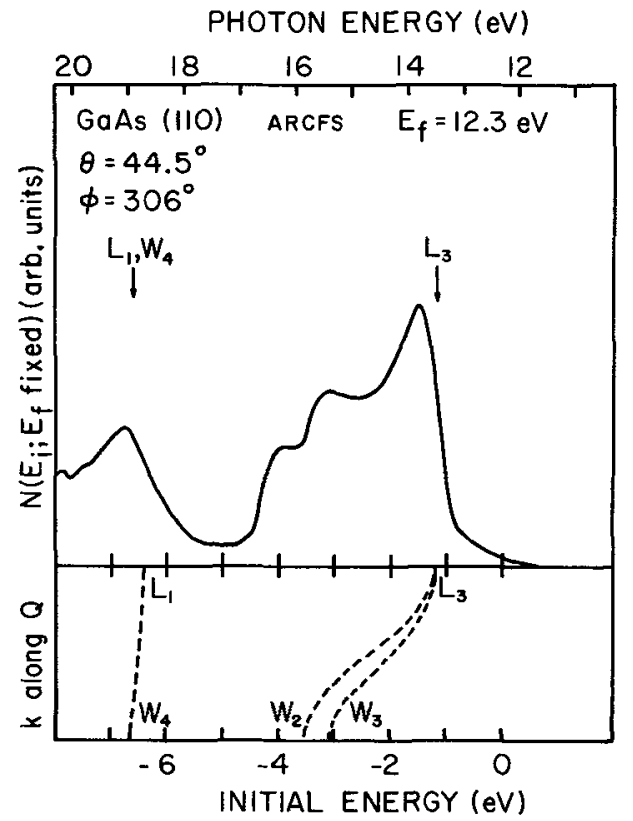

FIG. 3. - ARCFS from cleaved GaAs (110), taken for $\mathbf{k}_{\|}$at the $Q$ line, exhibiting VB emission from bands along $\mathrm{L}$ to $\mathrm{W}$.

$\langle 111\rangle$ azimuth. It shows maximum emission amplitude near the band edge $L_{3}$, about $1.15 \mathrm{eV}$ below the VB maximum. The remaining structure is attributed to the bands near $\mathrm{W}$ and $\mathrm{L}$, but detailed assignment requires a more complete set of ARCFS's as well as some idea of the final state band behaviour. By adjusting the final energy window $E_{\mathrm{f}}$ and emission angle $\theta_{p}$, one can maintain $\mathbf{k}_{\|}$constant and study the behaviour of the initial state bands along the symmetry line. This method has been applied to a study of the D line on $\mathrm{W}(001)[8,9]$.

The ARCFS mode of photoemission has also been used in recent studies of nickel [30] which revealed d-like initial state structure $4.3 \mathrm{eV}$ below the Fermi energy, $E_{\mathrm{F}}$. Among the band width studies of transition metals, the width of the $\mathrm{Ni} \mathrm{d}$-band, as determined by angle-integrated experiments, has been somewhat of an anomaly, since the measured width was significantly narrower than that of band calculations [31]. Our measured width, using PARUPS, 
is reasonably close to the calculated value. We show in figure 4 an ARCFS for the $Q$ line of $N i$ (110) recorded for a final energy of $17.8 \mathrm{eV}$ above $E_{\mathrm{F}}$. At this final energy, the structure at $-4.3 \mathrm{eV}$ is near its deepest $E_{\mathrm{i}}$ excursion. Examination of the calculated band structure [32] suggests that the transition responsible for this peak originates near $\mathrm{L}_{1}$. As $E_{\mathrm{f}}$ and $\theta_{p}$ are changed, maintaining $\mathbf{k}_{\|}$constant, the transition moves to smaller $E_{\mathrm{i}}$ values, consistent with the upward dispersion of the lowest band from $L_{1}$ to $W$. We can further verify the existence of this transition at $\mathrm{L}$ by examining data from a $\mathrm{Ni}(001)$ surface with $\mathbf{k}_{\|}$chosen to pass through the $\mathrm{L}$ point, i.e. the $\mathbf{k}_{\|}$line has a different direction which is now perpendicular to the (001) plane. The lower curve in figure 4 is an AREDC obtained from $\mathrm{Ni}(001)$

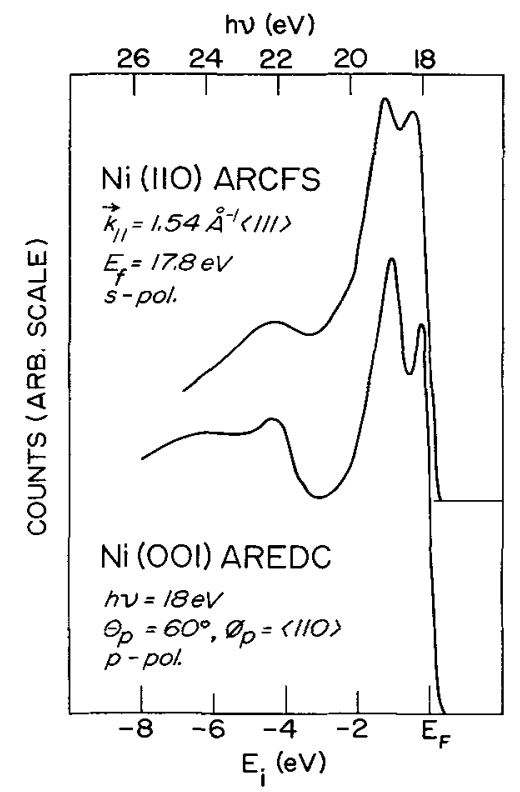

Fig. 4. - ARCFS from Ni (110), taken for $\mathbf{k}_{\|}$at the $Q$ line (upper curve) and an AREDC from $\mathrm{Ni}$ (001) exhibiting a transtion at $-4.3 \mathrm{eV}$ having $\mathbf{k}_{\|}$at $\mathrm{L}_{\|}$.

with $\theta_{p}=60^{\circ}, \varphi_{p}=\langle 110\rangle$ and $h v=18 \mathrm{eV}$. A transition is observed with $E_{\mathrm{i}}=-4.3 \mathrm{eV}$ which corresponds to $\mathbf{k}_{\|}$line passing through the $\mathrm{L}$ point. The similarity in energetics and amplitude for the $-4.3 \mathrm{eV}$ peak observed on both surfaces suggests that both peaks have a common source point in the BZ, specifically at L. Polarization dependent studies of this deep feature have shown that it is an even parity initial state, consistent with the $L_{1}$ assignment. The amplitude of this peak is relatively constant as a function of $E_{\mathrm{i}}$ and $k$ throughout the zone, which may explain why an angle averaged measurement reveals no structure at these initial energies, the peak being averaged over a range of $E_{\mathrm{i}}[30,33]$.

3. Core emission studies. - We have discussed how the three modes of photoemission can have unique applications in the study of valence and conduction band structure. Emission from deeper lying core levels represents still another area for which the ARCIS can have special utility since the initial energy window for the ARCIS spectrum is a constant, and the localized core levels have constant $E_{\mathrm{i}}$. With the ARCIS one can examine conduction band structure which provides final states above threshold, as well as special effects that occur near core excitation threshold, e.g. excitons.

3.1 Conduction BAND STUDIES USING CORE EMISSION. - An example of conduction band structure in a core level ARCIS is shown in figure 5. The upper

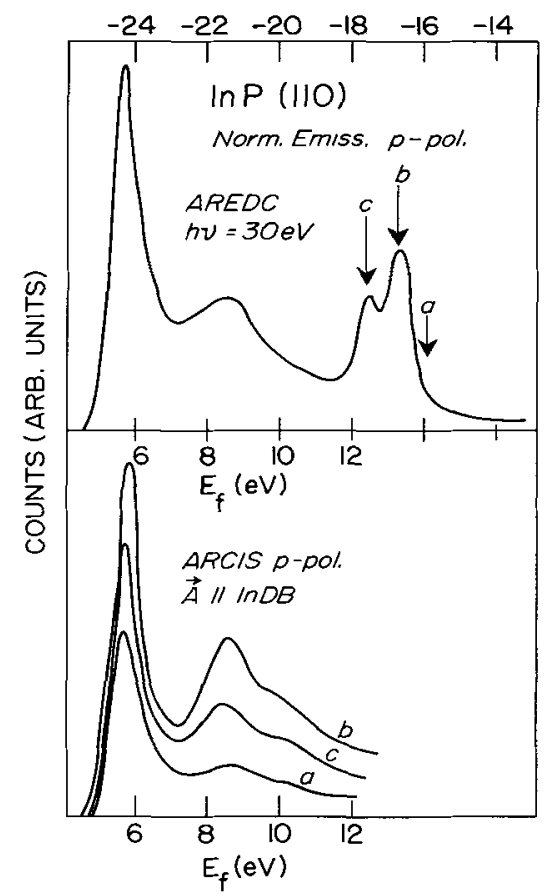

FIG. 5. - Normal emission AREDC from cleaved InP (110) exhibiting the split In $4 \mathrm{~d}$ core level and a replicated conduction band DOS (upper curve). Normal emission ARCIS's for In $4 d$ core emission showing a conduction band DOS at $E_{\mathrm{f}}=8.6 \mathrm{eV}$ (lower curves).

curve is an AREDC obtained at normal emission from cleaved InP (110) for $h v=30 \mathrm{eV}$. The spin-orbit split In $4 \mathrm{~d}$ core is observed with a splitting of $0.8 \mathrm{eV}$. The series of ARCIS spectra shown in the lower part of figure 5 were obtained with the initial energy window set just off the core (curve a) and on each member of the spin-orbit split pair (curves $b$ and $c$ ). Each core level goes through a maximum emission amplitude at a final energy $E_{\mathrm{f}}=8.6 \mathrm{eV}$ above the VB maximum. In addition, this conduction band feature is replicated by the inelastically scattered electrons [7, 28, 34] of the $30 \mathrm{eV}$ AREDC. We have measured emission from the VB maximum of InP [35], analogous to the measurements on GaAs shown in figure 1, and find that the final state at $8.6 \mathrm{eV}$, observed in the core level ARCIS of InP, is well below the $\Gamma_{12}$ final state, and may be due to the $\Gamma_{1}$ conduction band. 
The conduction band feature observed here is not seen in our angle integrated measurements, which further supports the interpretation of the normal emission structure as being due to a conduction band feature along $\Sigma$. Another point pertinent to the InP conduction band feature is that EDC's obtained for the. In $4 \mathrm{~d}$ core using resonant lamp radiation (e.g. Ne II at $h v=26.9 \mathrm{eV}$ ) could give misleading values for the statistical ratio of the split core levels since one core member would couple to the large final state density, while the other member would not. This potential problem points out an advantage of using the synchrotron continuum.

3.2 Photoemission enhancement at CORE thresHOLD. - A relationship between core excitation threshold and photocurrent yields has been reported by several researchers, e.g. Haensel et al. [2], and Gudat et al. [36]. Because of the work function barrier, direct core emission at threshold is not possible so the observed enhanced level of emission is due to decay processes, for example core-hole decay. Hence two facets of the enhanced emission need to be considered : 1) the photoabsorption process which generates the core holes, and 2) the non-radiative decay processes of the holes. Our development of the CFS and CIS methods was for the purpose of studying with UPS the reports of core enhancements in the alkali halides [2], which in most cases is due to excitons. (We observed enhanced emission in $\mathrm{Bi}_{2} \mathrm{Te}_{3}$ resulting from density of states structure in core to conduction band excitations [18].) However, we select, for presentation in this paper, $\mathrm{Ga} 3 \mathrm{~d}$ core threshold studies on the III-V semiconductor GaAs since the studies manifest a wide variety of phenomena including those reported for the alkali halides [3-6]. Enhanced photoemission for the $3 \mathrm{~d}$ core of $\mathrm{Ge}$ and $\mathrm{GaAs}$ was reported by Eastman and Freeouf and attributed to core-to-empty-surfacestate transitions [12]. While they found contamination sensitivity for the effect in Ge, they did not report such sensitivity for GaAs [37]. Our studies of the enhanced emission for GaAs indicated that the absorption process is due to exciton formation [11].

The core-to-surface-state exciton lines for GaAs 3d transitions as manifested in PARUPS CIS's are shown in figure 6. Examination of a family of CIS's shows that the exciton doublets are photon energy peaks being observed at particular $h v$ values while the interband transition structures to the left have final state character. The normal emission spectra are shown because the incident radiation vector can be rotated azimuthally while maintaining fixed geometry for the emission direction. As one notes in figure 6 , the exciton has strong polarization dependence, having strongest emission with respect to the azimuthal angle, $\varphi_{A}$, when $\mathbf{A}$ is in the GaAs (110) mirror plane and in the direction of the Ga Dangling Bond (DB). The Ga DB azimuth defines $\varphi=0^{\circ}$. The exciton lines are stronger for « $p$ » polarization than for s-pola-

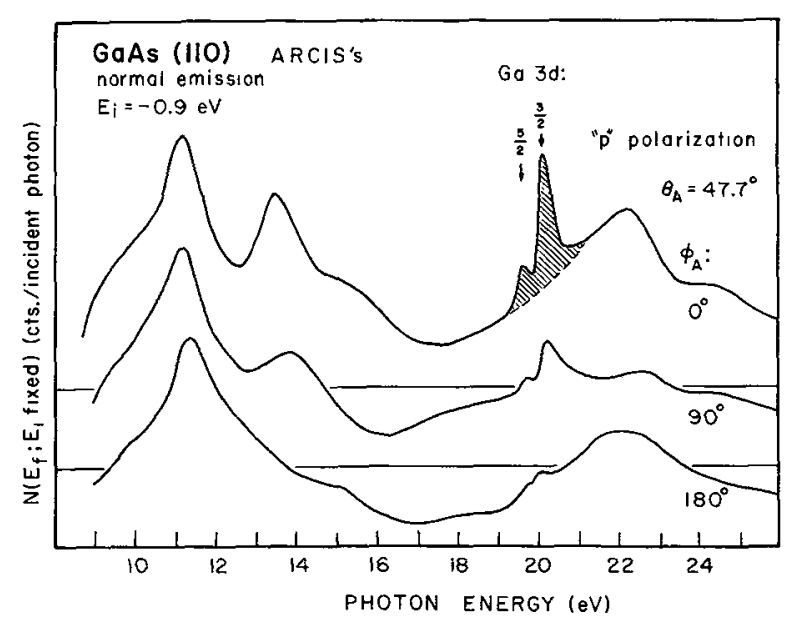

FIG. 6. - Normal emission ARCIS's from cleaved GaAs (110) exhibiting the polarization dependent surface exciton resonant emission. The cross-hatched area represents the exciton enhanced emission.

rization [11]. For « $\mathrm{p} »$ polarization, $\theta_{A}=47.7^{\circ}$ and quotation marks are put on « $\mathrm{p}$ » since our experimental geometry for this case contains about $50 \%$ s-polarization. Only $\theta_{A}$ of zero and $47.7^{\circ}$ have been measured. The observation that the emission is stronger when the incident $\mathbf{A}$ vector is nearly parallel to the $\mathrm{Ga} \mathrm{DB}$ has been used to support the assignment of the phenomenon to the Ga empty surface state, but a general argument that explains the polarization effect has not been constructed.

The most suggestive evidence for the exciton being a surface state effect is its contamination sensitivity, and an example of the sensitivity is shown in figure 7. The CMA angle integrated exciton lines are compared with the valence surface state labeled $B_{1}$ which is discussed elsewhere [38]. One notes essentially the same surface sensitivity for the exciton lines and for $B_{1}$. The wide peak above the exciton lines in the CFS is contamination insensitive and is attributed

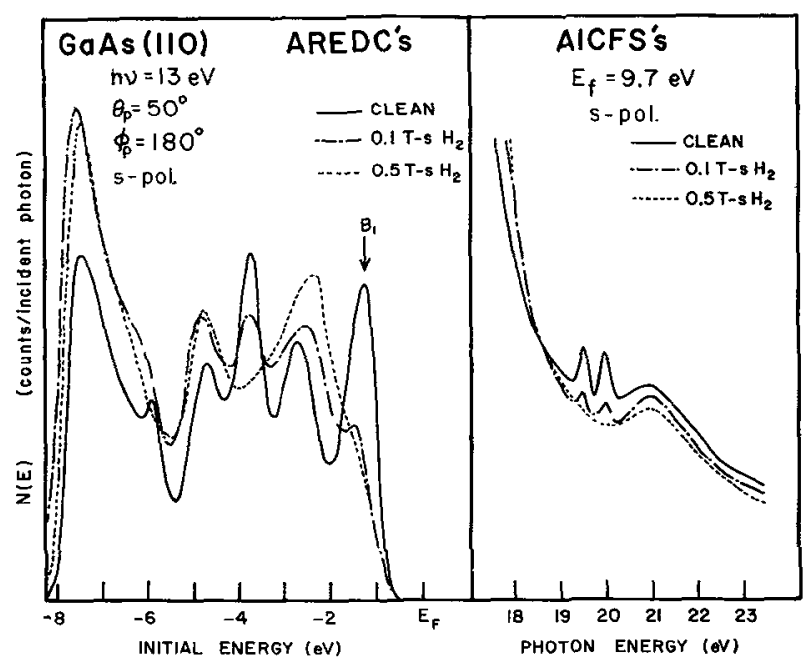

Fig. 7. - Contamination tests for the exciton resonance emission and for the $B_{1}$ surface state on cleaved GaAs (110). 
by Eastman and Freeouf [12] to enhancement resulting from core transitions to conduction band states. The latter peak and the exciton lines are superimposed on a large background of secondary electrons which have the same origin as the emission peak in an EDC near zero kinetic energy. The contamination sensitive peak at $E_{\mathbf{i}}=-3.8$ is tentatively assigned to a surface resonance recently found in surface state calculations [39]. Since the reproducibility of the peak for different cleaves was difficult, and no theoretical evidence for such a state existed, the peak's observation was suppressed in reference [38]. We have recently observed the analogous peak in InP and it shows contamination sensitivity similar to the GaAs case.

In order to study the core-hole-decay mechanisms that produce the enhanced emission, one would like the energy distribution for only the enhanced emission. While such a distribution is not directly measurable, it may be inferred from the measured emission. We propose two methods for determining the enhanced distribution. Both methods just give a representation for the enhanced distribution and since the methods depend on measuring and analyzing a number of spectra, the methods are subject to some uncertainty. Panel $\mathrm{C}$ in figure 8 shows our results for GaAs. The large dots represent the results of one method and were obtained from a set of CMA angle integrated CIS's in which the exciton peak to background ratio was formed for each CIS and then plotted against $E_{\mathrm{i}}$.

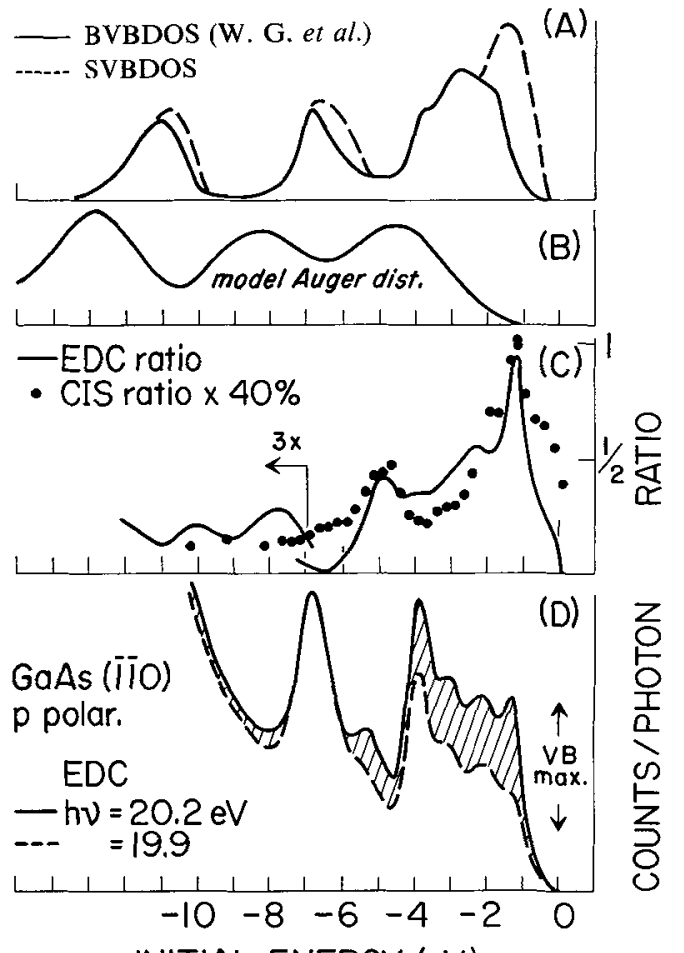

INITIAL ENERGY (eV)

FIG. 8. - Energy distribution of the exciton resonant enhancement on cleaved GaAs (110) obtained by two methods (panel C), compared with the VB DOS [41] (panel A), and its self convolution (panel B). EDC's taken at photon energies in the enhancement region (panel D).
The background was determined by eye as exemplified by the shading in figure 6 . Emission resulting from interband transitions is suppressed by this method. The other method is to form a ratio from two EDC's which are referenced to initial energy to suppress interband transitions. The result of the latter method is shown as the solid line in figure 8 panel $\mathrm{C}$ and the EDC's used are shown in the lower panel $D$. The EDC's were obtained at photon energies corresponding to the $j=3 / 2$ peak and the valley between the spin-orbit-split peaks. Both methods produce the same major features.

To account for the enhanced spectral features between $E_{\mathrm{i}}=-6 \mathrm{eV}$ and the $\mathrm{VB}$ maximum, we appeal to two non-radiative core-hole-decay processes $[2,3]$ which take place via the Coulomb interaction [40]. First is the Auger process whose final state is two valence band holes and an excited electron. Assuming no matrix element effects, the Auger electron distribution is given by the self-convolution of the VB density of states VBDOS and such a calculation based on a calculated [41] density of states is shown in panel B of figure 8. The peak at $E_{\mathrm{i}}=-5 \mathrm{eV}$ in the enhanced distribution (panel $C$ ) is attributed to the most energetic Auger peak.

The decay process, frequently called direct recombination, is the other mechanism we use to describe the enhanced distribution [2,3]. In direct recombination, the final state for the core-hole decay is a VB hole and an excited electron. The decay is due to Coulomb interaction $[3,40]$. In the absence of matrix element effects, the distribution of excited electrons is given by the simple VB density of states. Calculated bulk density of states as shown in panel $\mathrm{A}$, figure 8 , are used as a model for the direct recombination bulk distribution. The bulk VB density of states does not provide a good description for the enhanced distribution between the VB maximum and the Auger distribution; however, if surface VB density of states are included, reasonable agreement is obtained. Hence the strong narrow peak near the VB maximum in the enhanced distribution is attributed to the surface state which is labeled $B_{1}$ in figure 7 . We schematically show the surface density of states (Ref. [38]) as dashed lines in figure 8 , panel $\mathrm{A}$. The CFS for the contamination test (Fig. 7) was chosen at $E_{\mathrm{f}}=7.6 \mathrm{eV}$ so that both Auger and direct recombination electrons were collected by the spectrometer's energy window.

The existence of the direct recombination process is the primary evidence that leads to the interpretation of the enhancement peaks as excitons. That is, the strong interaction of the electron-hole pair comprising the exciton is intimately associated with the interactions leading to the direct recombination process. A very strong supporting argument for the exciton interpretation is the reversal of the relative line intensities of the spin-orbit pair from the $6: 4$ statistical weight for the core states. This reversal has a direct explanation in exciton theory [42]. 
Of particular interest is the oscillator strength and binding energy (greater than $0.45 \mathrm{eV}$ ) of the exciton enhancement as manifested in the direct recombination process. The exciton enhancement observed in GaAs and other III-V semiconductors is several orders of magnitude larger than for bulk excitons in semiconductors, due chiefly to three factors : 1) the extremely narrow width of the intrinsic surface state band out of which the exciton is drawn; 2) the reduced screening of the electron-hole interaction near the dangling bonds; and 3 ) the lower dimensionality of the system, involving the two-dimensional surface state. Gudat and Eastman [43] have used the CIS mode of photoemission to study the trends in excitonic binding energy for several III-V semiconductors.

Acknowledgment. - The efforts of P. L. Gobby and G. P. Williams are greatly appreciated as are useful communications with $\mathbf{J}$. Hermanson. It is a pleasure to acknowledge the assistance of $\mathrm{E}$. Rowe, Director of the Synchrotron Radiation Centre, and the services offered by $R$. Otte and the rest of the staff at SRC.

\section{References}

[1] The first report of the three modes was given in October, 1973, at the annual Wisconsin Synchrotron Radiation Centre users meeting; LAPEYRE, G. J., GoBby, P. L., KNAPP, J. A. and Anderson, J. R., Notes on the Sixth Annual Synchrotron Radiation Users Group Conference (1973) 22.

[2] Haensel, R., Keitel, G., Peters, G., Schreiber, P., Sonntag, B. and Kunz, C., Phys. Rev. Lett. 23 (1969) 530.

[3] Lapeyre, G. J., Baer, A. D., Hermanson, J., Anderson, J., Knapp, J. A., Gobby, P. L., Solid State Commun. 15 (1974) 1601.

[4] Lapeyre, G. J., Anderson, J., Knapp, J. A. and Gobby, P. L., in Vacuum Ultraviolet Radiation Physics, eds. E. E. Koch, R. Haensel and C. Kunz (Braunschweig : PergamonVieweg), 1974, 380;

ANDERSON, J. and LAPEYre, G. J., ibid. 404.

[5] Lapeyre, G. J., Anderson, J., Gobby, P. L. and KnapP, J. A., Phys. Rev. Lett. 33 (1974) 1290.

[6] Gobby, P. L. and Lapeyre, G. J., Bull. Am. Phys. Soc. 20 (1975) 474.

[7] Smith, R. J., Anderson, J., Hermanson, J. and Lapeyre, G. J., Solid State Commun. 19 (1976) 975.

[8] Lapeyre, G. J., Smith, R. J. and Anderson, J., J. Vac. Sci. Technol. 14 (1977) 384.

[9] Smith, R., Anderson, J., Lapeyre, G. and Hermanson, J., Bull. Am. Phys. Soc. 21 (1976) 431.

[10] Hermanson, J., Anderson, J. and Lapeyre, G., Phys. Rev. B 12 (1975) 5410.

[11] Lapeyre, G. J. and Anderson, J., Phys. Rev. Lett. 35 (1975) 117.

[12] Eastman, D. E. and Freeouf, J. L., Phys. Rev. Lett. 33 (1974) 1601.

[13] Gudat, W., Eastman, D. E. and Freeouf, J. L., J. Vac. Sci. Technol. 13 (1976) 250.

[14] KNAPP, J. A. and LAPEYRe, G. J., To be published in Nuovo Cimento ;

KnAPP, J. A., Thesis, Montana State University (1976), Dissertation Abstracts, Univ. Microfilms, Ann Arbor, MI.

[15] Gobby, P. L. and LaPeyre, G. J., in Physics of Semiconductors, ed. F. G. Fumi (Tipografia Marves, Rome) 1976, 150;

Gobby, P. L., Thesis, Montana State Univ. (1977), Dissertation Abstracts, Univ. Microfilms, Ann Arbor, MI.

[16] Bauer, R. S., Bachrach, R. Z., Flodstrom, S. A. and MCMenamin, J. C., J. Vac. Sci. Technol. 14 (1977) 378.

[17] Spicer, W. E., Lindau, I., Gregory, P. E., Garner, C. M., Pianetta, P. and Chye, P. W., J. Vac. Sci. Technol. 13 (1976) 780 .
[18] Hurych, Z., Shaffer, J. C., Davis, D. L., Knecht, T. A. LAPEYRE, G. J., GobBY, P. L., Knapp, J. A. and Olson, C. G., Phys. Rev. Lett. 33 (1974) 830.

[19] Margaritondo, G., Rowe, J. E. and Christman, S. B., Phys. Rev. B 15 (1977) 3844.

[20] Plummer, E. W., Gustaffson, T., Gudat, W. and Eastman, D. E., Phys. Rev. A 15 (1977) 2339.

[21] HuRych, Z. and Benbow, R. L., Phys. Rev. Lett. 38 (1977) 1094.

[22] Anderson, J., Lapeyre, G. J. and Smith, R. J., To be published in Phys. Rev. B.

[23] Weeks, S. P., J. Vac. Sci. Technol. 14 (1977) 480 ;

Weeks, S. P. and Plummer, E. W., Solid State Commun. 21 (1977) 695.

[24] Smith, N. V., Traum, M. M., Knapp, J. A., Anderson, J. and Lapeyre, G. J., Phys. Rev. B 13 (1976) 4462.

[25] Kane, E. O., Phys. Rev. Lett. 12 (1964) 97.

[26] The authors of reference [12] use the term partial yield for a CFS.

[27] LeE, P. A., Phys. Rev. B 13 (1976) 5261.

[28] Feuerbacher, B. and Christensen, N. E., Phys. Rev. B 10 (1974) 2373.

[29] Pandey, K. C. and Phillips, J. C., Phys. Rev. B 9 (1974) 1552.

[30] Smith, R. J., Anderson, J., Hermanson, J. and Lapeyre, G. J., Solid State Commun. 21 (1977) 459.

[31] McAlister, A. J., Curhill, J. R., Dobbyn, R. C., Williams, M. L. and Watson, R. E., Phys. Rev. B 12 (1975) 2973.

[32] Callaway, J. and Wang, C. S., Phys. Rev. B 7 (1973) 1096.

[33] Smith, R. J., Lapeyre, G. J. and Anderson, J., to appear in Physics of Transition Metals proceedings, Toronto (1977).

[34] Baer, A. D. and Lapeyre, G. J., Phys. Rev. Lett. 31 (1973) 304.

[35] Knapp, J. A. and Lapeyre, G. J., Bull. Am. Phys. Soc. 22 (1977) 363.

[36] Gudat, W. and Kunz, C., Phys. Rev. Lett. 29 (1972) 169.

[37] Eastman, D. E. and Freeouf, J. L., Phys. Rev. Lett. 34 (1975) 1624.

[38] KNapp, J. A. and Lapeyre, G. J., J. Vac. Sci. Technol. 13 (1976) 757.

[39] Pandey, K. C., Freeouf, J. L. and Eastman, D. E., J. Vac. Sci. Technol. 14 (1977) 904.

[40] Rubloff, G. W., Phys. Rev. B 5 (1972) 662.

[41] Eastman, D. E., Grobman, W. D., Frfeouf, J. L. and ErbuDAK, M., Phys. Rev. B 9 (1974) 3473.

[42] Onodera, Y. and Toyozawa, Y., J. Phys. Soc. Japan 22 (1967) 833.

[43] Gudat, W. and Eastman, D. E., J. Vac. Sci. Technol. 13 (1976) 831 . 